\title{
Determining the Robot-to-Robot 3D Relative Pose using Combinations of Range and Bearing Measurements (Part II)
}

\author{
Xun S. Zhou and Stergios I. Roumeliotis
}

\begin{abstract}
In this paper, we address the problem of motioninduced 3D robot-to-robot extrinsic calibration based on different combinations of inter-robot measurements (i.e., distance and/or bearing observations) and ego-motion estimates, recorded across multiple time steps. In particular, we focus on solving minimal problems where the unknown 6-degreeof-freedom transformation between two robots is determined based on the minimum number of measurements necessary for finding a discrete set of solutions. In our previous work [1], we have shown that only 14 base systems need to be solved, and provided closed-form solutions for three of them. This paper considers the remaining systems and provides closed-form solutions to most of them, while for some of the most challenging problems, we introduce efficient symbolic-numerical solution methods. Finally, we evaluate the performance of our proposed solvers through extensive simulations.
\end{abstract}

\section{INTRODUCTION AND RELATED WORK}

In order for robots to cooperate efficiently, they need to share information about their surroundings. Their measurements, however, are registered with respect to each robot's local reference frame and need to be converted to a common frame before they can be fused. This requires knowledge of the robot-to-robot transformation, i.e., their relative position and orientation (pose). To date, only few works describe how this transformation can be determined.

Manually measuring the relative pose between robots is obviously too tedious and time consuming. Alternatively, external references (e.g., GPS, compass, or maps of the environment) can be used to determine their relative pose. However, these external references are not always available (e.g., underwater, underground, outer space, or indoors).

In the absence of external references, the relative robotto-robot transformation can be computed using inter-robot observations, i.e., robot-to-robot distance and/or bearing measurements. For example, for the case of a static sensor network, numerous methods have been proposed for determining the locations of sensors in 2D using distance measurements between neighboring sensors (e.g., [2]). Moreover, flying anchors have been used to localize ground sensors, e.g., an aerial vehicle aiding static sensor network localization [3], or a single satellite localizing a stationary planetary rover [4]. However, all these methods only determine the positions of static sensors.

For mobile sensors, the problem of relative pose determination has only been studied thoroughly in $2 \mathrm{D}$. In particular, the ability to move and collect measurements from

This work was supported by the University of Minnesota (DTC), and the National Science Foundation (IIS-0643680, IIS-0811946, IIS-0835637).

Xun S. Zhou and Stergios I. Roumeliotis are with the Department of Computer Science and Engineering, University of Minnesota, Minneapolis, MN, 55455 USA email: $\{$ zhoulstergios $\}$ eds. umn.edu different vantage points provides additional information for localizing the sensors, and hence makes their relative pose observable [5]. Specifically, it is known that mutual distance and bearing measurements between two robots from a single vantage point are sufficient for determining the 3-degrees-offreedom (DOF) relative transformation in closed-form [6], [7]. However, when only distance or bearing measurements are available, the robots must move and record additional observations. Then the relative robot pose can be found by combining the estimated robot motion (e.g., from odometry) with the mutual bearing [5] or distance [8] measurements.

In contrast to the case of motion in $2 \mathrm{D}$, the more challenging problem of determining relative pose in $3 \mathrm{D}$ has received limited attention. Interestingly, the task of relativepose estimation using only 6 distance measurements is actually equivalent to the forward-kinematics problem of the general Stewart-Gough platform [9]. This problem has 40 (generally complex) solutions [10], which can be found by solving a system of multivariate polynomial equations [11], [12]. Moreover, in our previous work [13] we presented methods for estimating the robots' relative pose when both robots measure relative distance and bearing, or bearing only. However, to the best of our knowledge, no algorithms exist for determining 3D relative pose using different combinations of robot-to-robot distance and/or bearing measurements over time (e.g., the robots can measure distance at the first time step, bearing at the second time step, etc.). Our recent publication [1] and this paper intend to fill this gap.

Specifically, in [1], we focused on solving minimal problems where the number of equations, provided by the interrobot measurements, equals the number of unknown parameters. In particular, we considered the case where the robots are equipped with different types of sensors, or record different types of relative measurements over time due to environment constraints. Such minimal problems are formulated as systems of polynomial equations which, in general, have multiple (complex) solutions. We have identified 14 base minimal systems (see Fig. 2), based on which all other combinations can be solved, and provided closedform solutions to three of them (Systems 1, 2, and 5). In this paper, we will discuss the remaining minimal systems and provide closed-form or symbolic-numeric solutions for each one. The solutions to these minimal problems are very useful in practice for two reasons: i) in the presence of measurement outliers, using minimal solvers as hypothesis generators minimizes the number of samples required in an outlier-rejection scheme such as Random Sample Consensus (RANSAC), ii) minimal solvers can be used to initialize an iterative process [e.g., nonlinear weighted least squares 


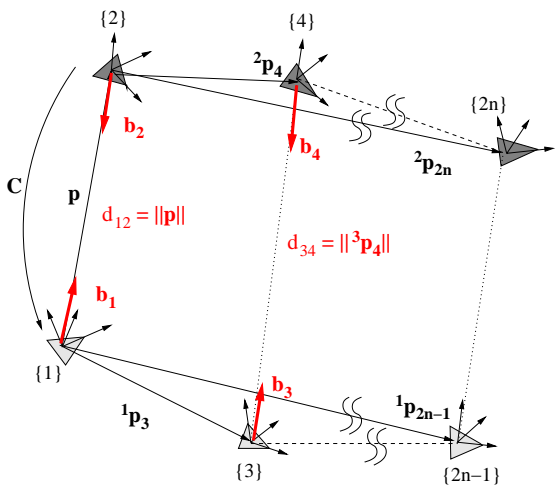

(a)

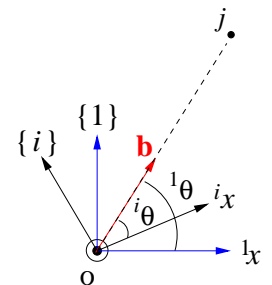

(b)
Fig. 1. (a) Geometry of the robot trajectories. The odd (even) numbered frames of reference depict the consecutive poses of robot $R_{1}\left(R_{2}\right)$. The distance between the robot poses $\{i\}$ and $\{j\}$ is denoted by $d_{i j}, i \in$ $\{1,3, \ldots, 2 n-1\}, j \in\{2,4, \ldots, 2 n\} . \mathbf{b}_{i}\left(\mathbf{b}_{j}\right)$ is a unit vector pointing from $\{i\}$ to $\{j\}$ ( $\{j\}$ to $\{i\})$ expressed in the initial frame $\{1\}(\{2\})$. The problem is to determine the transformation between the robots' initial frames $\{1\}$ and $\{2\}$, parametrized by the translation vector $\mathbf{p}$ and the rotation matrix C. (b) Illustration of a bearing measurement expressed in different frames of reference. The bearing $\mathbf{b}$ is a unit vector pointing from $o$ to $j$. When expressed in frame $\{i\}$, the angle between $\mathbf{b}$ and the $x$-axis of frame $\{i\},{ }^{i} x$, is ${ }^{i} \theta$. When expressed in frame $\{1\}$, the angle between $\mathbf{b}$ and the $x$-axis of frame $\{1\},{ }^{1} x$, is ${ }^{1} \theta$. Transforming the bearing from $\{i\}$ to $\{1\}$ is done by multiplying with the rotation matrix ${ }_{i}^{1} \mathbf{C}: \mathbf{b}_{i}={ }_{i}^{1} \mathbf{C}^{i} \mathbf{b}_{j}$.

(NWLS)] for improving the estimation accuracy when additional measurements are available ${ }^{1}$.

The remainder of the paper is organized as follows: After describing the problem formulation in Section II, we present closed-form solutions to Systems 6 and 7 in Section III. Then, we solve Systems $8-10$ by using the Macaulay resultant [14] in Section IV, and solve Systems 11-13 by applying Reid and Zhi's [15] symbolic-numeric algorithm in Section V. The accuracy of the presented methods is evaluated through Monte-Carlo simulations in Section VI, followed by our concluding remarks and future work in Section VII.

\section{Problem Formulation}

The following notation is used in this paper:

${ }^{i} \mathbf{p}_{j} \quad$ Position of frame $\{j\}$ expressed in frame $\{i\}$.

${ }_{j}^{i} \mathbf{C}$ Rotation matrix that projects vectors expressed in frame $\{j\}$ to frame $\{i\}$.

$d_{i j} \quad$ Distance between the origin of frame $\{i\}$ and $\{j\}$.

$\mathbf{b}_{i} \quad$ The bearing from robot $R_{1}$ to $R_{2}$ when $R_{1}$ is at pose $\{i\}=\{2 n-1\}, n \in \mathbb{N}^{*}$, expressed in frame $\{1\}$.

$\mathbf{b}_{j} \quad$ The bearing from robot $R_{2}$ to $R_{1}$ when $R_{2}$ is at pose $\{j\}=\{2 n\}, n \in \mathbb{N}^{*}$, expressed in frame $\{2\}$.

$s \alpha \quad$ Short for $\sin (\alpha)$.

$c \alpha \quad$ Short for $\cos (\alpha)$.

Consider two robots $R_{1}$ and $R_{2}$ moving randomly in 3D through a sequence of poses $\{1\},\{3\}, \ldots,\{2 n-1\}$ for $R_{1}$, and $\{2\},\{4\}, \ldots,\{2 n\}$ for $R_{2}$ [see Fig. 1(a)]. Along

${ }^{1}$ Note also that an additional advantage of NWLS is that it accounts for the effect of uncertainty and noise in the available motion estimates and inter-robot measurements, respectively.

\begin{tabular}{|c|c|c|c|c|c|c|c|}
\hline & $t_{1}$ & $t_{2}$ & $t_{3}$ & $t_{4}$ & $t_{5}$ & $t_{6}$ & $\mathrm{r}$ \\
\hline 1 & $d_{12}, \mathbf{b}_{1}, \mathbf{b}_{2}$ & $d_{34}$ & & & & & 2 \\
\hline 2 & $\mathbf{b}_{1}, \mathbf{b}_{2}$ & $\mathbf{b}_{3}$ & & & & & 2 \\
\hline 3 & $d_{12}, \mathbf{b}_{1}$ & $d_{34}, \mathbf{b}_{3}$ & & & & & $\infty$ \\
\hline 4 & $d_{12}, \mathbf{b}_{1}$ & $d_{34}, \mathbf{b}_{4}$ & & & & & $\infty$ \\
\hline 5 & $\mathbf{b}_{1}, \mathbf{b}_{2}$ & $d_{34}$ & $d_{56}$ & & & & 4 \\
\hline 6 & $d_{12}, \mathbf{b}_{1}$ & $\mathbf{b}_{3}$ & $d_{56}$ & & & & 4 \\
\hline 7 & $d_{12}, \mathbf{b}_{1}$ & $\mathbf{b}_{4}$ & $d_{56}$ & & & & 4 \\
\hline 8 & $\mathbf{b}_{1}$ & $\mathbf{b}_{3}$ & $\mathbf{b}_{5}$ & & & & 8 \\
\hline 9 & $\mathbf{b}_{1}$ & $\mathbf{b}_{3}$ & $\mathbf{b}_{6}$ & & & & 8 \\
\hline 10 & $d_{12}, \mathbf{b}_{1}$ & $d_{34}$ & $d_{56}$ & $d_{78}$ & & & 8 \\
\hline 11 & $\mathbf{b}_{1}$ & $\mathbf{b}_{3}$ & $d_{56}$ & $d_{78}$ & & & 16 \\
\hline 12 & $\mathbf{b}_{1}$ & $\mathbf{b}_{4}$ & $d_{56}$ & $d_{78}$ & & & 16 \\
\hline 13 & $\mathbf{b}_{1}$ & $d_{34}$ & $d_{56}$ & $d_{78}$ & $d_{9,10}$ & & 28 \\
\hline 14 & $d_{12}$ & $d_{34}$ & $d_{56}$ & $d_{78}$ & $d_{9,10}$ & $d_{11,12}$ & 40 \\
\hline
\end{tabular}

Fig. 2. 14 minimal problems. Under column $t_{i}$ are measurements recorded at time step $i$. The last column (under $r$ ) shows the number of solutions to each system. The problems not covered in this paper are colored in gray. Systems 1, 2, and 5 are solved in [1]. Systems 3 and 4 have infinite solutions as shown in [16]. System 14 is addressed in [10], [11], [12].

their trajectories, the robots can estimate their positions ${ }^{1} \mathbf{p}_{i}$ and ${ }^{2} \mathbf{p}_{j}, i \in\{1,3, \ldots, 2 n-1\}, j \in\{2,4, \ldots, 2 n\}$, with respect to their initial frames, as well as their orientations, represented by the rotation matrices ${ }_{i}^{1} \mathbf{C}$ and ${ }_{j}^{2} \mathbf{C}$ (e.g., by integrating linear and rotational velocity measurements over time). Additionally, at time-step $t_{n}$ when robots $R_{1}$ and $R_{2}$ reach poses $\{i=2 n-1\}$ and $\{j=2 n\}$, respectively, each robot can measure the range and/or bearing towards the other robot. The range between the robots is given by $d_{i j}=\left\|{ }^{i} \mathbf{p}_{j}\right\|_{2}$, and the bearing is described by a unit vector in the current local frame ${ }^{i} \mathbf{b}_{j}$ for robot $R_{1}$ and ${ }^{j} \mathbf{b}_{i}$ for robot $R_{2}$. Later on, we will also need these unit vectors expressed in the robots' initial frames and thus we define $\mathbf{b}_{i}:={ }_{i}^{1} \mathbf{C}^{i} \mathbf{b}_{j}$ and $\mathbf{b}_{j}:={ }_{j}^{2} \mathbf{C}^{j} \mathbf{b}_{i}$ [see Fig. 1(b)]. At each time step, the two robots can measure a subset of these measurements: $\left\{d_{i j}, \mathbf{b}_{i}, \mathbf{b}_{j}\right\}$.

Our goal is to use the ego-motion estimates and the relative pose measurements to determine the 6-DOF initial transformation between the two robots, i.e., their relative position $\mathbf{p}:={ }^{1} \mathbf{p}_{2}$ and orientation $\mathbf{C}:={ }_{2}^{1} \mathbf{C}$. In this paper, we only focus on solving the minimal problems where the number of measurement constraints equals the number of unknowns (see Fig. 2). In our previous work [1], we provided solutions for Systems 1, 2, and 5. Since Systems 3 and 4 have infinite solutions [16] and System 14 has been extensively studied (e.g., [10], [11], [12]), we will present analytic or numerical solutions for the remaining systems (Systems 613) in the following sections.

\section{Closed-Form SOlutions FOR Systems 6 AND 7}

Due to their similarities, Systems 6 and 7 can be formulated as the same system of equations and solved using the same technique. In both systems, the relative position $\mathbf{p}=d_{12} \mathbf{b}_{1}$ is directly measured and the unknown quantities are in the rotation matrix $\mathbf{C}$. In the following sections, we first derive the system of equations for both problems, and then present their closed-form solution. 


\section{A. System 6: Measurements $\left\{d_{12}, \mathbf{b}_{1} ; \mathbf{b}_{3} ; d_{56}\right\}$}

Given the relative position $\mathbf{p}=d_{12} \mathbf{b}_{1}$ and the second bearing measurement $\mathbf{b}_{3}$, we have the following constraint, which is the sum of vectors from $\{1\}$ to $\{2\},\{4\},\{3\}$, and back to $\{1\}$ [see Fig. 1(a)]:

$$
\begin{aligned}
& \mathbf{p}+\mathbf{C} \cdot{ }^{2} \mathbf{p}_{4}-{ }_{3}^{1} \mathbf{C} \cdot{ }^{3} \mathbf{p}_{4}-{ }^{1} \mathbf{p}_{3}=0 \\
& \Rightarrow \mathbf{p}+\mathbf{C} \cdot{ }^{2} \mathbf{p}_{4}-d_{34} \mathbf{b}_{3}-{ }^{1} \mathbf{p}_{3}=0 .
\end{aligned}
$$

Next, we eliminate $d_{34}$ from (1) by forming dot products with vectors perpendicular to $\mathbf{b}_{3}$. Let $\mathbf{v}_{1}=\mathbf{b}_{3} \times\left(\mathbf{p}-{ }^{1} \mathbf{p}_{3}\right)$ and $\mathbf{v}_{2}=\mathbf{b}_{3} \times \mathbf{v}_{1}$. Projecting (1) onto $\mathbf{v}_{1}$ and $\mathbf{v}_{2}$ yields

$$
\begin{aligned}
\mathbf{v}_{1}^{T} \mathbf{C} \cdot{ }^{2} \mathbf{p}_{4} & =0 \\
\mathbf{v}_{2}^{T} \mathbf{C} \cdot{ }^{2} \mathbf{p}_{4}+\mathbf{v}_{2}^{T}\left(\mathbf{p}-{ }^{1} \mathbf{p}_{3}\right) & =0 .
\end{aligned}
$$

Finally, by expanding the constraint from the distance measurement $d_{56}$, we have the following third equation necessary for solving the 3-DOF relative orientation $\mathbf{C}$.

$$
\begin{gathered}
{ }^{5} \mathbf{p}_{6}^{T 5} \mathbf{p}_{6}=\left(\mathbf{p}+\mathbf{C}^{2} \mathbf{p}_{6}-{ }^{1} \mathbf{p}_{5}\right)^{T}\left(\mathbf{p}+\mathbf{C}^{2} \mathbf{p}_{6}-{ }^{1} \mathbf{p}_{5}\right)=d_{56}^{2} \\
\Rightarrow 2\left(\mathbf{p}-{ }^{1} \mathbf{p}_{5}\right)^{T} \mathbf{C} \cdot{ }^{2} \mathbf{p}_{6}+\epsilon=0
\end{gathered}
$$

where $\epsilon=\mathbf{p}^{T} \mathbf{p}+{ }^{2} \mathbf{p}_{6}^{T 2} \mathbf{p}_{6}+{ }^{1} \mathbf{p}_{5}^{T 1} \mathbf{p}_{5}-2 \mathbf{p}^{T 1} \mathbf{p}_{5}-d_{56}^{2}$.

\section{B. System 7: Measurements $\left\{d_{12}, \mathbf{b}_{1} ; \mathbf{b}_{4} ; d_{56}\right\}$}

Similar to System 6, we have the following constraint from the first three measurements.

$$
\begin{aligned}
\mathbf{p}+\mathbf{C} \cdot{ }^{2} \mathbf{p}_{4}+\mathbf{C} \cdot{ }_{4}^{2} \mathbf{C} \cdot{ }^{4} \mathbf{p}_{3}-{ }^{1} \mathbf{p}_{3} & =0 \\
\Rightarrow \mathbf{C}^{T}\left(\mathbf{p}-{ }^{1} \mathbf{p}_{3}\right)+{ }^{2} \mathbf{p}_{4}+d_{34} \mathbf{b}_{4} & =0 .
\end{aligned}
$$

Defining $\mathbf{v}_{1}=\mathbf{b}_{4} \times{ }^{2} \mathbf{p}_{4}$, and $\mathbf{v}_{2}=\mathbf{b}_{4} \times \mathbf{v}_{1}$, and forming dot products with (6), we have

$$
\begin{aligned}
\mathbf{v}_{1}^{T} \mathbf{C}^{T}\left(\mathbf{p}-{ }^{1} \mathbf{p}_{3}\right) & =0 \\
\mathbf{v}_{2}^{T} \mathbf{C}^{T}\left(\mathbf{p}-{ }^{1} \mathbf{p}_{3}\right)+\mathbf{v}_{2}^{T 2} \mathbf{p}_{4} & =0 .
\end{aligned}
$$

Together with the distance constraint (4), we have three equations for solving $\mathbf{C}$.

\section{Closed-form Solution for the Rotation Matrix}

For both Systems 6 and 7, we need to solve for the rotation matrix $^{2} \mathbf{C}$ from systems of equations of the form [see (2), (3), and (4) or see (7), (8), and (4)]

$$
\begin{aligned}
\mathbf{v}_{1}^{T} \mathbf{C} \mathbf{u}_{1} & =0 \\
\mathbf{v}_{2}^{T} \mathbf{C u}_{1}+w_{2} & =0 \\
\mathbf{v}_{3}^{T} \mathbf{C u}_{3}+w_{3} & =0
\end{aligned}
$$

where the $\mathbf{v}_{i}$ 's, $\mathbf{u}_{i}$ 's, $w_{i}$ 's are known quantities. In the following, we will describe an elimination procedure to solve this system of equations. Similar procedures have been applied in the past to determine camera pose using line-toplane correspondences [17].

From a geometric point of view, (9) is satisfied when vector $\mathbf{v}_{1}$ is perpendicular to the rotated $\mathbf{u}_{1}$. Therefore, we can determine one DOF in rotation which is the rotation around a unit vector $\mathbf{e}_{2}$ perpendicular to both $\mathbf{v}_{1}$ and $\mathbf{u}_{1}$ (see Fig. 3). Then the remaining two DOF in rotation are determined using the two constraints (10) and (11).

${ }^{2}$ For System 7 , we solve for $\mathbf{C}^{T}$, then the rotation matrix $\mathbf{C}$ is readily available as its transpose.

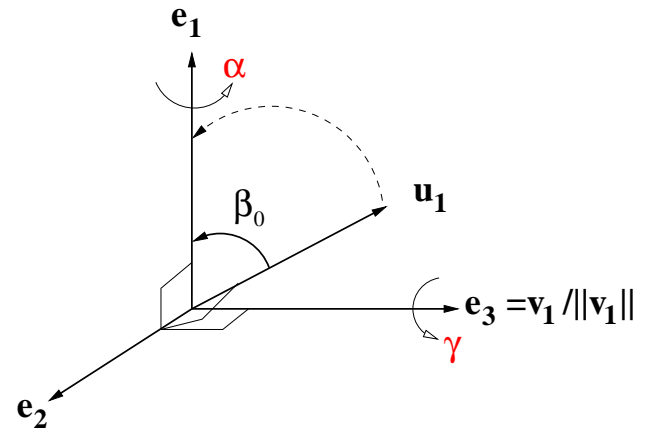

Fig. 3. Sequence of rotations that satisfies the constraint: $\mathbf{v}_{1}^{T} \mathbf{C} \mathbf{u}_{1}=0$. Let $\mathbf{e}_{3}=\frac{\mathbf{v}_{1}}{\left\|\mathbf{v}_{1}\right\|}, \mathbf{e}_{2}=\frac{\mathbf{v}_{1} \times \mathbf{u}_{1}}{\left\|\mathbf{v}_{1} \times \mathbf{u}_{1}\right\|}$, and $\mathbf{e}_{1}=\mathbf{e}_{2} \times \mathbf{e}_{3}$. Rotating around $\mathbf{e}_{2}$ by $\beta_{0}$ aligns $\mathbf{u}_{1}$ with $\mathbf{e}_{1}$ so that the constraint is satisfied for arbitrary rotations around the other two axes $\mathbf{e}_{1}$ and $\mathbf{e}_{3}$.

Specifically, we parametrize the rotation matrix $\mathbf{C}$ using three rotational angles $\gamma, \beta$, and $\alpha$ around axes $\mathbf{e}_{3}=\frac{\mathbf{v}_{1}}{\left\|\mathbf{v}_{1}\right\|}$, $\mathbf{e}_{2}=\frac{\mathbf{v}_{1} \times \mathbf{u}_{1}}{\left\|\mathbf{v}_{1} \times \mathbf{u}_{1}\right\|}$, and $\mathbf{e}_{1}=\mathbf{e}_{2} \times \mathbf{e}_{3}$, respectively. Without loss of generality, we assume that $\mathbf{e}_{1}, \mathbf{e}_{2}$, and $\mathbf{e}_{3}$ are unit vectors corresponding to the three principal axes ${ }^{3}$, i.e., $\mathbf{e}_{1}=\left[\begin{array}{lll}1 & 0 & 0\end{array}\right]^{T}$, etc. Thus, we write the rotation matrix as:

$$
\begin{aligned}
\mathbf{C} & =\mathbf{C}\left(\mathbf{e}_{3}, \gamma\right) \mathbf{C}\left(\mathbf{e}_{1}, \alpha\right) \mathbf{C}\left(\mathbf{e}_{2}, \beta\right) \\
& =\left[\begin{array}{ccc}
c \gamma & -s \gamma & 0 \\
s \gamma & c \gamma & 0 \\
0 & 0 & 1
\end{array}\right]\left[\begin{array}{ccc}
1 & 0 & 0 \\
0 & c \alpha & -s \alpha \\
0 & s \alpha & c \alpha
\end{array}\right]\left[\begin{array}{ccc}
c \beta & 0 & s \beta \\
0 & 1 & 0 \\
-s \beta & 0 & c \beta
\end{array}\right] .
\end{aligned}
$$

Substituting the above expression for $\mathbf{C}$ in (9), and using the fact that $\mathbf{v}_{1}$ aligns with the rotation axis $\mathbf{e}_{3}$, we have

$$
\begin{array}{r}
\mathbf{v}_{1}^{T} \mathbf{C}\left(\mathbf{e}_{3}, \gamma\right) \mathbf{C}\left(\mathbf{e}_{1}, \alpha\right) \mathbf{C}\left(\mathbf{e}_{2}, \beta\right) \mathbf{u}_{1}=0 \\
\Rightarrow \mathbf{v}_{1}^{T} \mathbf{C}\left(\mathbf{e}_{1}, \alpha\right) \mathbf{C}\left(\mathbf{e}_{2}, \beta\right) \mathbf{u}_{1}=0 .
\end{array}
$$

Note that (13) must be satisfied for any angle $\alpha$, else it will contradict (10) and (11) (see Fig. 3). Thus, after applying the first rotation, $\mathbf{u}_{1}$ must align with $\mathbf{e}_{1}$, i.e.,

$$
\mathbf{C}\left(\mathbf{e}_{2}, \beta\right) \mathbf{u}_{1}= \pm \mathbf{e}_{1}\left\|\mathbf{u}_{1}\right\| \text {. }
$$

Hereafter, we choose the solution with the positive $\operatorname{sign}^{4}$. Specifically, from (14), we can determine $\beta=\beta_{0}$,

$$
c \beta_{0}=\mathbf{u}_{1}^{T} \mathbf{e}_{1} /\left\|\mathbf{u}_{1}\right\|, \quad s \beta_{0}=\mathbf{u}_{1}^{T} \mathbf{e}_{3} /\left\|\mathbf{u}_{1}\right\| .
$$

The remaining unknown angles $\alpha$ and $\gamma$ are determined from (10) and (11) by first substituting $\beta_{0}$ and treating (temporarily) $s \alpha$ and $c \alpha$ as known. This yields a linear system of equations which we solve to determine $s \gamma$ and $c \gamma$ as functions of $s \alpha$ and $c \alpha$. Then using the trigonometric constraints $s \gamma^{2}+c \gamma^{2}=1$ and $s \alpha^{2}+c \alpha^{2}=1$, we derive a $4^{\text {th }}$ order univariate polynomial in $s \alpha$.

Specifically, let $\mathbf{v}_{2}=\left[\begin{array}{lll}v_{2 x} & v_{2 y} & v_{2 z}\end{array}\right]^{T}$. From (10), we have

$$
\begin{aligned}
& \mathbf{v}_{2}^{T} \mathbf{C}\left(\mathbf{e}_{3}, \gamma\right) \mathbf{C}\left(\mathbf{e}_{1}, \alpha\right) \mathbf{C}\left(\mathbf{e}_{2}, \beta_{0}\right) \mathbf{u}_{1}+w_{2} \\
= & \left(v_{2 x} c \gamma+v_{2 y} s \gamma\right)\left\|\mathbf{u}_{1}\right\|+w_{2}=0 .
\end{aligned}
$$

From (11) we have

$$
\begin{aligned}
& \mathbf{v}_{3}^{T} \mathbf{C}\left(\mathbf{e}_{3}, \gamma\right) \mathbf{C}\left(\mathbf{e}_{1}, \alpha\right) \mathbf{C}\left(\mathbf{e}_{2}, \beta_{0}\right) \mathbf{u}_{3}+w_{3} \\
= & l_{1} c \gamma+l_{2} s \gamma+l_{3}=0
\end{aligned}
$$

\footnotetext{
${ }^{3}$ The solution to the general case where $\mathbf{e}_{1}, \mathbf{e}_{2}$, and $\mathbf{e}_{3}$ form any other orthonormal basis is described in [16].

${ }^{4}$ The negative-sign solution leads to exactly the same four solutions for the relative pose. Due to space limitations, we omit its derivation.
} 
where $l_{1}, l_{2}$, and $l_{3}$, whose exact expressions are given in [16], are linear functions of $s \alpha$ and $c \alpha$.

From (16) and (17), we can solve for $c \gamma$ and $s \gamma$ linearly.

$$
\left[\begin{array}{l}
c \gamma \\
s \gamma
\end{array}\right]=\frac{-1}{v_{2 x} l_{2}-v_{2 y} l_{1}}\left[\begin{array}{l}
l_{2} w_{2}^{\prime}-v_{2 y} l_{3} \\
v_{2 x} l_{3}-l_{1} w_{2}^{\prime}
\end{array}\right]
$$

where $w_{2}^{\prime}=w_{2} /\left\|\mathbf{u}_{1}\right\|$.

Substituting the above expression for $c \gamma$ and $s \gamma$ in the trigonometric constraint $c \gamma^{2}+s \gamma^{2}=1$, we obtain a quadratic polynomial in $c \alpha$ and $s \alpha$ (embedded in $l_{1}, l_{2}$, and $l_{3}$ ).

$$
\begin{aligned}
& \left(l_{2} w_{2}^{\prime}-v_{2 y} l_{3}\right)^{2}+\left(v_{2 x} l_{3}-w_{2}^{\prime} l_{1}\right)^{2}=\left(v_{2 x} l_{2}-v_{2 y} l_{1}\right)^{2} \\
& \Rightarrow k_{1} c \alpha^{2}+\left(k_{2} s \alpha+k_{4}\right) c \alpha+k_{3} s \alpha^{2}+k_{5} s \alpha+k_{6}=0
\end{aligned}
$$

where the coefficients $k_{1}, \ldots, k_{6}$ are functions of known quantities [16].

Finally, using the trigonometric constraint $s \alpha^{2}+c \alpha^{2}=1$, we employ the Sylvester resultant to eliminate $c \alpha$ from (19), and obtain a $4^{\text {th }}$ order univariate polynomial in $s \alpha$. The Sylvester resultant is the determinant of the matrix:

$\left[\begin{array}{cccc}k_{1} & k_{2} s \alpha+k_{4} & k_{3} s \alpha^{2}+k_{5} s \alpha+k_{6} & 0 \\ 0 & k_{1} & k_{2} s \alpha+k_{4} & k_{3} s \alpha^{2}+k_{5} s \alpha+k_{6} \\ 1 & 0 & s \alpha^{2}-1 & 0 \\ 0 & 1 & 0 & s \alpha^{2}-1\end{array}\right]$

which must be equal to zero, i.e.,

$$
r_{1} s \alpha^{4}+r_{2} s \alpha^{3}+r_{3} s \alpha^{2}+r_{4} s \alpha+r_{5}=0 .
$$

where $r_{1}, \ldots, r_{5}$ are functions of $k_{1}, \ldots, k_{6}$.

Hence, there are 4 solutions for $s \alpha$. Back-substituting each solution for $s \alpha$ into (19), we compute the corresponding solutions for $c \alpha$ :

$$
c \alpha=-\frac{\left(k_{3}-k_{1}\right) s \alpha^{2}+k_{5} s \alpha+k_{1}+k_{6}}{k_{2} s \alpha+k_{4}} .
$$

where we have substituted $c \alpha^{2}$ with $1-s \alpha^{2}$. Finally, backsubstituting the 4 solutions of $s \alpha$ and $c \alpha$ into the linear equation (18), we compute the corresponding 4 solutions for $c \gamma$ and $s \gamma$. Therefore, there are a total of 4 solutions for the relative pose for Systems 6 and 7 .

\section{Symbolic-Numerical SOLUTIONS FOR Systems 8,9 , AND 10}

These three problems are more challenging, since we can no longer exploit geometric properties to solve for the unknown DOF separately. However, these systems share the same algebraic structure that allows us to find their solutions via an algebraic-geometry method, based on the Macaulay resultant [14]. In the following, we first derive the system of equations for these three problems, and then present the Macaulay resultant based solutions.

\section{A. System 8: Measurements $\left\{\mathbf{b}_{1} ; \mathbf{b}_{3} ; \mathbf{b}_{5}\right\}$}

From Fig. 1(a), using measurements $\mathbf{b}_{1}$ and $\mathbf{b}_{3}$, and $\mathbf{b}_{1}$ and $\mathbf{b}_{5}$, we have the following two constraints:

$$
\begin{aligned}
& d_{12} \mathbf{b}_{1}+\mathbf{C} \cdot{ }^{2} \mathbf{p}_{4}-d_{34} \mathbf{b}_{3}-{ }^{1} \mathbf{p}_{3}=0 \\
& d_{12} \mathbf{b}_{1}+\mathbf{C} \cdot{ }^{2} \mathbf{p}_{6}-d_{56} \mathbf{b}_{5}-{ }^{1} \mathbf{p}_{5}=0
\end{aligned}
$$

whose difference gives:

$$
d_{34} \mathbf{b}_{3}+\mathbf{C}\left({ }^{2} \mathbf{p}_{6}-{ }^{2} \mathbf{p}_{4}\right)-d_{56} \mathbf{b}_{5}-\left({ }^{1} \mathbf{p}_{5}-{ }^{1} \mathbf{p}_{3}\right)=0 .
$$

If the rotation matrix $\mathbf{C}$ was known, we can solve for $d_{12}$ by first eliminating the unknown $d_{34}$ from (22) by forming the cross product with $\mathbf{b}_{3}$ :

$$
d_{12}\left\lfloor\mathbf{b}_{3} \times\right\rfloor \mathbf{b}_{1}+\left\lfloor\mathbf{b}_{3} \times\right\rfloor \mathbf{C} \cdot{ }^{2} \mathbf{p}_{4}-\left\lfloor\mathbf{b}_{3} \times\right\rfloor{ }^{1} \mathbf{p}_{3}=\mathbf{0}
$$

where $\left\lfloor\mathbf{b}_{3} \times\right\rfloor$ is the $3 \times 3$ skew-symmetric matrix corresponding to the cross product. Then $d_{12}$ can be computed from (25) by forming the dot product with $\left\lfloor\mathbf{b}_{3} \times\right\rfloor \mathbf{b}_{1}$, i.e.,

$$
d_{12}=\frac{\left(\left\lfloor\mathbf{b}_{3} \times\right\rfloor \mathbf{b}_{1}\right)^{T}\left\lfloor\mathbf{b}_{3} \times\right\rfloor\left({ }^{1} \mathbf{p}_{3}-\mathbf{C} \cdot{ }^{2} \mathbf{p}_{4}\right)}{\left(\left\lfloor\mathbf{b}_{3} \times\right\rfloor \mathbf{b}_{1}\right)^{T}\left(\left\lfloor\mathbf{b}_{3} \times\right\rfloor \mathbf{b}_{1}\right)} .
$$

Next, we describe how to eliminate all the unknown distances, $d_{12}, d_{34}$, and $d_{56}$, from (22), (23), and (24), so that we can solve for C. Specifically, we define three vectors

$$
\mathbf{v}_{1}=\mathbf{b}_{1} \times \mathbf{b}_{3}, \quad \mathbf{v}_{2}=\mathbf{b}_{1} \times \mathbf{b}_{5}, \quad \mathbf{v}_{3}=\mathbf{b}_{3} \times \mathbf{b}_{5}
$$

and form their dot products with (22), (23), and (24), respectively:

$$
\begin{aligned}
\mathbf{v}_{1}^{T} \mathbf{C} \cdot{ }^{2} \mathbf{p}_{4}-\mathbf{v}_{1}^{T 1} \mathbf{p}_{3} & =0 \\
\mathbf{v}_{2}^{T} \mathbf{C} \cdot{ }^{2} \mathbf{p}_{6}-\mathbf{v}_{2}^{T 1} \mathbf{p}_{5} & =0 \\
\mathbf{v}_{3}^{T} \mathbf{C}\left({ }^{2} \mathbf{p}_{6}-{ }^{2} \mathbf{p}_{4}\right)-\mathbf{v}_{3}^{T}\left({ }^{1} \mathbf{p}_{5}-{ }^{1} \mathbf{p}_{3}\right) & =0 .
\end{aligned}
$$

From (28)-(30), we determine C (see Section IV-D).

\section{B. System 9: Measurements $\left\{\mathbf{b}_{1} ; \mathbf{b}_{3} ; \mathbf{b}_{6}\right\}$}

Systems 9 and 8 both contain measurements $\mathbf{b}_{1}$ and $\mathbf{b}_{3}$. Therefore, System 9 also contains equation (28). The differences lie in the next two constraints using $\mathbf{b}_{1}$ and $\mathbf{b}_{6}$, and $\mathbf{b}_{3}$ and $\mathbf{b}_{6}$ :

$$
\begin{aligned}
d_{12} \mathbf{b}_{1}+\mathbf{C} \cdot{ }^{2} \mathbf{p}_{6}+d_{56} \mathbf{C b}_{6}-{ }^{1} \mathbf{p}_{5} & =0 \\
d_{34} \mathbf{b}_{3}+\mathbf{C}\left({ }^{2} \mathbf{p}_{6}-{ }^{2} \mathbf{p}_{4}\right)+d_{56} \mathbf{C b}_{6}-\left({ }^{1} \mathbf{p}_{5}-{ }^{1} \mathbf{p}_{3}\right) & =0
\end{aligned}
$$

Similarly to System 8, we define

$$
\mathbf{v}_{1}=\mathbf{b}_{1} \times \mathbf{b}_{3}, \quad \mathbf{v}_{2}=\mathbf{b}_{1} \times \mathbf{C b}_{6}, \quad \mathbf{v}_{3}=\mathbf{b}_{3} \times \mathbf{C b}_{6}
$$

and project equation (31) to $\mathbf{v}_{2}$ to obtain

$$
\begin{aligned}
&\left(\mathbf{b}_{1} \times \mathbf{C b}_{6}\right)^{T} \mathbf{C}^{2} \mathbf{p}_{6}-{ }^{1} \mathbf{p}_{5}^{T}\left(\mathbf{b}_{1} \times \mathbf{C b}_{6}\right)=0 \\
& \Rightarrow \mathbf{b}_{1}^{T}\left(\mathbf{C b}_{6} \times \mathbf{C}^{2} \mathbf{p}_{6}\right)-\left({ }^{1} \mathbf{p}_{5} \times \mathbf{b}_{1}\right)^{T} \mathbf{C b}_{6}=0 \\
& \Rightarrow \mathbf{b}_{1}^{T} \mathbf{C}\left(\mathbf{b}_{6} \times{ }^{2} \mathbf{p}_{6}\right)-\left({ }^{1} \mathbf{p}_{5} \times \mathbf{b}_{1}\right)^{T} \mathbf{C b}_{6}=0
\end{aligned}
$$

where from (34) to (35), we have applied the identity ( $\mathbf{a} \times$ $\mathbf{b})^{T} \mathbf{c}=\mathbf{a}^{T}(\mathbf{b} \times \mathbf{c})$.

Similarly projecting (32) to $\mathbf{v}_{3}$, yields

$$
\mathbf{b}_{3}^{T} \mathbf{C u}_{3}-\mathbf{u}_{2}^{T} \mathbf{C b}_{6}=0
$$

where $\mathbf{u}_{3}=\mathbf{b}_{6} \times\left({ }^{2} \mathbf{p}_{6}-{ }^{2} \mathbf{p}_{4}\right)$, and $\mathbf{u}_{2}=\left({ }^{1} \mathbf{p}_{5}-{ }^{1} \mathbf{p}_{3}\right) \times$ $\mathbf{b}_{3}$. Finally, from (28), (36), and (37), we solve for $\mathbf{C}$ (see Section IV-D). Note also that, $d_{12}$ is computed from (26).

\section{System 10: Measurements $\left\{d_{12}, \mathbf{b}_{1} ; d_{34} ; d_{56} ; d_{78}\right\}$}

In this problem, the relative position is known from $\mathbf{p}=d_{12} \mathbf{b}_{1}$. The remaining quantity to be determined is the rotation matrix $\mathbf{C}$. From the three distance measurements $d_{2 i-1,2 i}, i=2,3,4$, we have [see (4)]:

$$
2\left(\mathbf{p}-{ }^{1} \mathbf{p}_{2 i-1}\right)^{T} \mathbf{C} \cdot{ }^{2} \mathbf{p}_{2 i}+\epsilon_{i}=0, i=2,3,4
$$

where $\epsilon_{i}=\mathbf{p}^{T} \mathbf{p}+{ }^{2} \mathbf{p}_{2 i}^{T}{ }^{2} \mathbf{p}_{2 i}+{ }^{1} \mathbf{p}_{2 i-1}^{T}{ }^{1} \mathbf{p}_{2 i-1}-2 \mathbf{p}^{T 1} \mathbf{p}_{2 i-1}-$ $d_{2 i-1,2 i}^{2}$. 
In summary, Systems 8,9 , and 10 share the same form of equations for solving $\mathbf{C}$, i.e.,

$$
f_{i}=\sum \mathbf{v}^{T} \mathbf{C u}+w_{i}=0, \quad i=1,2,3 .
$$

If we use Cayley's parameters to express

$$
\mathbf{C}=\frac{\left(1-\mathbf{s}^{T} \mathbf{s}\right) \mathbf{I}+2\lfloor\mathbf{s} \times\rfloor+2 \mathbf{s s}^{T}}{1+\mathbf{s}^{T} \mathbf{s}}
$$

then (39) becomes a system of quadratic polynomial equations in three unknowns $\mathbf{s}=\left[s_{1}, s_{2}, s_{3}\right]^{T}$. The Bézout bound of the maximum number of solutions is $2^{3}=8$ for this system. We can show that the bound is reached generically by any instances of the problem. When the Bézout bound is reached, we can apply the Macaulay resultant [14] to solve systems of polynomial equations.

\section{Application of the Macaulay Resultant}

In order to construct the Macaulay resultant matrix, we need to add a linear polynomial $f_{0}$ to the system (39):

$$
\left\{\begin{array}{l}
f_{0}=1+s_{1}+s_{2}+s_{3} \\
f_{i}=\sum \mathbf{v}^{T} \mathbf{C u}+w_{i}, i=1,2,3
\end{array}\right.
$$

Then we take the monomials up to total degree

$$
t=\sum_{i=0}^{n} t_{i}-n=1+2+2+2-3=4
$$

where $t_{i}$ is the total degree of $f_{i}$ and $n$ is the number of variables. So there are a total of $\left(\begin{array}{c}4+3 \\ 3\end{array}\right)=35$ monomials in $s_{1}, s_{2}$, and $s_{3}$, which we divide into four sets, $S_{0}, \ldots, S_{3}$.

The basis monomials, $S_{0}$, are

$$
S_{0}=\left\{s_{1} s_{2} s_{3}, s_{1} s_{2}, s_{1} s_{3}, s_{2} s_{3}, s_{1}, s_{2}, s_{3}, 1\right\},
$$

while following [14] $S_{1}, S_{2}$, and $S_{3}$ are defined as:

$$
\begin{aligned}
S_{1}= & \left\{s_{1}^{4}, s_{1}^{3} s_{2}, s_{1}^{3} s_{3}, s_{1}^{2} s_{2} s_{3}, s_{1}^{3}, s_{1}^{2} s_{2}, s_{1}^{2} s_{3}, s_{1}^{2}\right\} \\
S_{2}= & \left\{s_{1}^{2} s_{2}^{2}, s_{1} s_{2}^{3}, s_{2}^{4}, s_{1} s_{2}^{2} s_{3}, s_{2}^{3} s_{1}, s_{1} s_{2}^{2}, s_{2}^{3}, s_{2}^{2} s_{3}, s_{2}^{2}\right\} \\
S_{3}= & \left\{s_{1}^{2} s_{3}^{2}, s_{1} s_{2} s_{3}^{2}, s_{2}^{2} s_{3}^{2}, s_{1} s_{3}^{3}, s_{2} s_{3}^{3}, s_{3}^{4}, s_{1} s_{3}^{2},\right. \\
& \left.s_{2} s_{3}^{2}, s_{3}^{3}, s_{3}^{2}\right\}
\end{aligned}
$$

where $S_{3}$ contains monomials that can be divided by $s_{3}^{2}, S_{2}$ contains monomials that can be divided by $s_{2}^{2}$ but not $s_{3}^{2}, S_{1}$ contains monomials that can be divided by $s_{1}^{2}$ but not $s_{2}^{2}$ and $s_{3}^{2}$, and $S_{0}$ contains the remaining monomials.

The Macaulay matrix $\mathbf{M}$ is constructed by multiplying $f_{0}$ with monomials in $S_{0}$, and multiplying $f_{i}$ with monomials in $S_{i}$ divided by $s_{i}^{2}, i=1,2,3$. This gives us a total of 35 equations. Arranging them in a matrix form $\mathbf{M} \underline{\mathbf{s}}=\mathbf{0}$, where $\underline{\mathbf{S}}$ is the vector containing all the 35 monomials, yields the Macaulay matrix:

$$
\mathbf{M}=\left[\begin{array}{ll}
\mathbf{M}_{00} & \mathbf{M}_{01} \\
\mathbf{M}_{10} & \mathbf{M}_{11}
\end{array}\right]
$$

where each row contains the coefficients of a polynomial. Each column of $\mathbf{M}$ corresponds to one of the 35 monomials. In particular, $\mathbf{M}_{00}$ is a $8 \times 8$ matrix whose columns correspond to the 8 monomials in $S_{0}$, and $\mathbf{M}_{01}$ is a $8 \times 27$ matrix whose columns correspond to the rest of the monomials. The rows of $\mathbf{M}_{00}$ and $\mathbf{M}_{01}$ correspond to the 8 polynomials resulting from multiplying $f_{0}$ with monomials in $S_{0}$, while $\mathbf{M}_{10}$ and $\mathbf{M}_{11}$ correspond to the remaining 27 polynomials resulting from multiplying $f_{i}$ with appropriate monomials.
Taking the Schur complement of $\mathbf{M}$, yields the multiplication matrix

$$
\mathbf{M}_{f_{0}}=\mathbf{M}_{00}-\mathbf{M}_{01} \mathbf{M}_{11}^{-1} \mathbf{M}_{10} .
$$

The eigenvectors $\boldsymbol{\xi}_{i}$ of $\mathbf{M}_{f_{0}}, i=1, \ldots, 8$, are vectors whose elements are monomials in $S_{0}$ evaluated at the 8 solutions. Therefore, we can read off the solutions for $s_{1}, s_{2}, s_{3}$. Note that these are the $5^{\text {th }}, 6^{\text {th }}$, and $7^{\text {th }}$ elements of $\boldsymbol{\xi}_{i}$, $i=1, \ldots, 8$, after proper scaling, i.e.,

$$
s_{1}=\boldsymbol{\xi}_{i}(5) / \boldsymbol{\xi}_{i}(8), s_{2}=\boldsymbol{\xi}_{i}(6) / \boldsymbol{\xi}_{i}(8), s_{3}=\boldsymbol{\xi}_{i}(7) / \boldsymbol{\xi}_{i}(8) .
$$

\section{Symbolic-Numerical SOlutions FOR Systems}

$$
11,12 \text {, AND } 13
$$

For Systems 11-13, it is very challenging to find closedform solutions. Also, attempts to apply the Macaulay resultant fail, because the number of solutions to these systems is less than the Bézout bound making $\mathbf{M}_{11}$ singular. Fortunately, recent progress in algebraic geometry has provided a symbolic-numerical method for constructing the multiplication matrix for any system. In the following sections, we derive the polynomial equations for Systems 11, 12, and 13, and then present the symbolic-numerical method [15] we employed for solving these systems.

\section{A. System 11: Measurements $\left\{\mathbf{b}_{1} ; \mathbf{b}_{3} ; d_{56} ; d_{78}\right\}$}

For this problem, we first write the unknown $d_{12}$ as a function of the rotation matrix $\mathbf{C}$. Then by substituting it into other constraints, we form a system of polynomials only in the rotation parameters. Even though the total degree of the resulting system is higher than before eliminating $d_{12}$, it reduces the problem size and makes the computation faster, as we will see later.

Specifically, from the bearing measurements $\mathbf{b}_{1}$ and $\mathbf{b}_{3}$ [see (22)], we eliminate the unknown $d_{34}$ and solve for $d_{12}$ [see (26)]:

$$
d_{12}=\mathbf{v}_{1}^{T} \mathbf{C} \cdot{ }^{2} \mathbf{p}_{4}+a
$$

where

$$
\mathbf{v}_{1}^{T}=-\frac{\left(\left\lfloor\mathbf{b}_{3} \times\right\rfloor \mathbf{b}_{1}\right)^{T}\left\lfloor\mathbf{b}_{3} \times\right\rfloor}{\left(\left\lfloor\mathbf{b}_{3} \times\right\rfloor \mathbf{b}_{1}\right)^{T}\left(\left\lfloor\mathbf{b}_{3} \times\right\rfloor \mathbf{b}_{1}\right)}, \quad a=-\mathbf{v}_{1}^{T 1} \mathbf{p}_{3} .
$$

We then substitute $d_{12}$ [see (45)] into the following two distance constraints:

$$
\begin{aligned}
& \left(\mathbf{p}+\mathbf{C} \cdot{ }^{2} \mathbf{p}_{6}-{ }^{1} \mathbf{p}_{5}\right)^{T}\left(\mathbf{p}+\mathbf{C} \cdot{ }^{2} \mathbf{p}_{6}-{ }^{1} \mathbf{p}_{5}\right)=d_{56}^{2} \\
& \left(\mathbf{p}+\mathbf{C} \cdot{ }^{2} \mathbf{p}_{8}-{ }^{1} \mathbf{p}_{7}\right)^{T}\left(\mathbf{p}+\mathbf{C} \cdot{ }^{2} \mathbf{p}_{8}-{ }^{1} \mathbf{p}_{7}\right)=d_{78}^{2} .
\end{aligned}
$$

The resulting equations are functions in only $\mathbf{C}$

$$
\begin{aligned}
& \left(\mathbf{v}_{1}^{T} \mathbf{C} \cdot{ }^{2} \mathbf{p}_{4}\right)+2\left(\mathbf{v}_{1}^{T} \mathbf{C} \cdot{ }^{2} \mathbf{p}_{4}\right)\left(\mathbf{b}_{1}^{T} \mathbf{C} \cdot{ }^{2} \mathbf{p}_{6}\right) \\
& \quad+2 \mathbf{b}_{1}^{T} \mathbf{u}_{1}\left(\mathbf{v}_{1}^{T} \mathbf{C} \cdot{ }^{2} \mathbf{p}_{4}\right)+2 \mathbf{u}_{1}^{T} \mathbf{C} \cdot{ }^{2} \mathbf{p}_{6}+\epsilon_{1}=0 \\
& \left(\mathbf{v}_{1}^{T} \mathbf{C} \cdot{ }^{2} \mathbf{p}_{4}\right)+2\left(\mathbf{v}_{1}^{T} \mathbf{C} \cdot{ }^{2} \mathbf{p}_{4}\right)\left(\mathbf{b} 1 \mathbf{C} \cdot{ }^{2} \mathbf{p}_{8}\right) \\
& \quad+2 \mathbf{b}_{1}^{T} \mathbf{u}_{2}\left(\mathbf{v}_{1}^{T} \mathbf{C} \cdot{ }^{2} \mathbf{p}_{4}\right)+2 \mathbf{u}_{2}^{T} \mathbf{C} \cdot{ }^{2} \mathbf{p}_{8}+\epsilon_{2}=0
\end{aligned}
$$

where

$$
\begin{array}{ll}
\mathbf{u}_{1}=a \mathbf{b}_{1}-{ }^{1} \mathbf{p}_{5}, & \epsilon_{1}={ }^{2} \mathbf{p}_{6}^{T 2} \mathbf{p}_{6}+\mathbf{u}_{1}^{T} \mathbf{u}_{1}-d_{56}^{2} \\
\mathbf{u}_{2}=a \mathbf{b}_{1}-{ }^{1} \mathbf{p}_{7}, & \epsilon_{2}={ }^{2} \mathbf{p}_{8}^{T 2} \mathbf{p}_{8}+\mathbf{u}_{2}^{T} \mathbf{u}_{2}-d_{78}^{2} .
\end{array}
$$

Finally, let $\mathbf{v}_{2}=\mathbf{b}_{1} \times \mathbf{b}_{3}$. Projecting (22) on $\mathbf{v}_{2}$ results in a third equation in $\mathbf{C}$

$$
\mathbf{v}_{2}^{T} \mathbf{C} \cdot{ }^{2} \mathbf{p}_{4}-\mathbf{v}_{2}^{T 1} \mathbf{p}_{3}=0 .
$$


Now, we have three equations (49)-(51) to solve for the 3DOF rotation matrix $\mathbf{C}$.

Using Cayley's parameter [see (40)] is not a good choice here, because it introduces spurious solutions corresponding to $1+\mathbf{s}^{T} \mathbf{s}=0$ when multiplying all equations with this factor to convert them into polynomials. Instead, we choose the (non-Hamiltonian) quaternion [18] to parametrize orientation defined as $\bar{q}=\left[\begin{array}{llll}q_{1} & q_{2} & q_{3} & q_{4}\end{array}\right]^{T}=\left[\begin{array}{ll}\mathbf{q}^{T} & q_{4}\end{array}\right]^{T}$, and related to the rotational matrix as ${ }^{5}$ :

$$
\mathbf{C}(\bar{q})=\mathbf{I}_{3}-2 q_{4}\lfloor\mathbf{q} \times\rfloor+2\lfloor\mathbf{q} \times\rfloor^{2} .
$$

Substituting (52) in (49) and (50) yields two $4^{\text {th }}$ order polynomials, while (51) becomes quadratic. Together with the unit-norm constraint

$$
\bar{q}^{T} \bar{q}-1=0
$$

forms a square system, which we can solve for $\bar{q}$.

\section{B. System 12: Measurements $\left\{\mathbf{b}_{1} ; \mathbf{b}_{4} ; d_{56} ; d_{78}\right\}$}

In this case, we cannot easily solve for $d_{12}$ and $\mathbf{C}$ separately, because monomials with both $d_{12}$ and elements of $\mathbf{C}$ exist in the system. Instead, we form a square system of polynomial equations in both $d_{12}$ and $\mathbf{C}$ using the available measurements which we solve based on the methods described in Section V-D.

Specifically, multiplying (5) by $\mathbf{C}^{T}$ yields

$$
d_{12} \mathbf{C}^{T} \mathbf{b}_{1}+{ }^{2} \mathbf{p}_{4}+d_{34} \mathbf{b}_{4}-\mathbf{C}^{T 1} \mathbf{p}_{3}=0 .
$$

Then we eliminate $d_{34}$ by forming the dot product with two unit vectors $\mathbf{v}_{1}$ and $\mathbf{v}_{2}$ both of which are perpendicular to $\mathbf{b}_{4}$, and obtain two equations:

$$
d_{12} \mathbf{v}_{i}^{T} \mathbf{C}^{T} \mathbf{b}_{1}-\mathbf{v}_{i}^{T} \mathbf{C}^{T 1} \mathbf{p}_{3}+\mathbf{v}_{i}^{T 2} \mathbf{p}_{4}=0, i=1,2 .
$$

Expanding the distance constraints (47) and (48), we have

$$
\begin{aligned}
& d_{12}^{2}+2 d_{12}\left(\mathbf{b}_{1}^{T} \mathbf{C}^{2} \mathbf{p}_{6}-\mathbf{b}_{1}^{T 1} \mathbf{p}_{5}\right)-2^{1} \mathbf{p}_{5}^{T} \mathbf{C}^{2} \mathbf{p}_{6}+\epsilon_{1}=0 \\
& d_{12}^{2}+2 d_{12}\left(\mathbf{b}_{1}^{T} \mathbf{C}^{2} \mathbf{p}_{8}-\mathbf{b}_{1}^{T 1} \mathbf{p}_{7}\right)-2^{1} \mathbf{p}_{7}^{T} \mathbf{C}^{2} \mathbf{p}_{8}+\epsilon_{2}=0
\end{aligned}
$$

where

$$
\epsilon_{1}={ }^{2} \mathbf{p}_{6}^{T 2} \mathbf{p}_{6}+{ }^{1} \mathbf{p}_{5}^{T 1} \mathbf{p}_{5}-d_{56}^{2}, \epsilon_{2}={ }^{2} \mathbf{p}_{8}^{T 2} \mathbf{p}_{8}+{ }^{1} \mathbf{p}_{7}^{T 1} \mathbf{p}_{7}-d_{78}^{2} .
$$

Equations (55)-(57) and the unit-norm constraint (53) form a square system of five polynomial equations allowing us to solve for $d_{12}$ and $\bar{q}$.

\section{System 13: Measurements $\left\{\mathbf{b}_{1} ; d_{34} ; d_{56} ; d_{78} ; d_{9,10}\right\}$}

From the bearing and distance measurements, we have the following constraints [see (56)], $i=2, \ldots, 5$

$$
d_{12}^{2}+2 d_{12}\left(\mathbf{b}_{1}^{T} \mathbf{C}^{2} \mathbf{p}_{2 i}-\mathbf{b}_{1}^{T 1} \mathbf{p}_{2 i-1}\right)-2{ }^{1} \mathbf{p}_{2 i-1}^{T} \mathbf{C}^{2} \mathbf{p}_{2 i}+\epsilon_{i}=0
$$

where $\epsilon_{i}={ }^{2} \mathbf{p}_{2 i}^{T}{ }^{2} \mathbf{p}_{2 i}+{ }^{1} \mathbf{p}_{2 i-1}^{T}{ }^{1} \mathbf{p}_{2 i-1}-d_{2 i-1,2 i}^{2}$. Together with the unit-norm constraint (53), we have a square system of five polynomial equations to solve for $d_{12}$ and $\bar{q}$.

\footnotetext{
${ }^{5}$ Note that since the quaternions $\bar{q}$ and $-\bar{q}$ both represent the same rotation, the number of solutions is double. One can easily eliminate the redundant solutions by discarding those with $q_{4}<0$.
}

\section{Symbolic-Numeric Solutions}

In this section, we briefly describe Reid and Zhi's symbolic-numeric method [15] for solving systems of polynomial equations and its application to Systems 11-13. This method also computes the solutions via the multiplication matrix, similar to the Macaulay resultant, but it is not limited to the case where the Bézout bound is reached.

Specifically, we expand the original systems of equations by multiplying them with monomials up to certain total degrees to form a new system

$$
\mathbf{M}_{t} \mathbf{x}_{t}=\mathbf{0}
$$

where $\mathbf{M}_{t}$ is a matrix containing all the coefficients of the expanded polynomial equations, and $\mathbf{x}_{t}$ is a vector of monomials up to total degree $t$. Therefore, the solutions of the system of polynomial equations must lie in the null space of $\mathbf{M}_{t}$. Unlike the Macaulay matrix where the total degree is determined by a specific formula [see (42)], the total degree here is computed via a so-called involutive-form test. This test also tells us how many solutions the system has.

The involutive test includes prolongation and projection operations. A single prolongation of a system $F$ means to expand it to one higher total degree. Prolongation up to total degree $t$ is denoted by $F^{(t)}$. After each prolongation, we compute the null space of the coefficient matrix $\mathbf{M}_{t}$. A single projection means to remove rows of the vectors spanning the null space of $\mathbf{M}_{t}$ such that they contain monomials with one lower total degree, i.e., $\mathbf{x}_{t}$ reduces to $\mathbf{x}_{t-1}$. One projection of a system $F$ is denoted by $\pi(F)$, and higher projection orders are denoted by $\pi^{i}(F), i=2, \ldots, t$.

A system $F$ is involutive at order $t$ and projection order $\ell$ if and only if $\pi^{\ell}\left(F^{(t)}\right)$ satisfies

$$
\operatorname{dim} \pi^{\ell}\left(F^{(t)}\right)=\operatorname{dim} \pi^{\ell+1}\left(F^{(t+1)}\right)=\operatorname{dim} \pi^{\ell+1}\left(F^{(t)}\right)
$$

where dim denotes the dimension of the corresponding space. Furthermore, $\operatorname{dim} \pi^{\ell}\left(F^{(t)}\right)$ equals the number of solutions of system $F$.

This involutive test only needs to be performed once. After we know the total degree $t$ to which to expand the system, we can use it to construct $\mathbf{M}_{t}$ for all instances of the problem. Then we compute the multiplication matrix with respect to an unknown variable, in our case $q_{4}$. In general, we can choose any variable as the multiplier as long as the solutions for this variable are distinct (see Ch. 2, Proposition 4.7 in [14]). For the specific problems at hand, we cannot choose $d_{12}$, because its solution multiplicity is at least two, since each solution for $d_{12}$ corresponds to solutions $\bar{q}$ and $-\bar{q}$. More detailed steps to compute the multiplication matrix are listed in the following:

1) Compute the null space of $\mathbf{M}_{t}$, so that the columns of matrix $\mathbf{B}_{t}$ span its null space.

2) Perform $\ell$ and $\ell-1$ projections on $\mathbf{B}_{t}$, i.e., take the rows of $\mathbf{B}_{t}$ that correspond to monomials up to total degree $t-\ell$ and $t-\ell-1$ to form the new matrices $\mathbf{B}$ and $\mathbf{B}_{1}$, respectively.

3) Compute the SVD of $\mathbf{B}_{1}=\left[\begin{array}{ll}\mathbf{U}_{1} & \mathbf{U}_{2}\end{array}\right] \cdot\left[\begin{array}{ll}\mathbf{S} & \mathbf{0}\end{array}\right]^{T} \cdot \mathbf{V}^{T}$.

4) Form the multiplication matrix of $q_{4}$ by $\mathbf{M}_{q_{4}}=$ $\mathbf{U}_{1}^{T} \mathbf{B}_{q_{4}} \mathbf{V S}^{-1}$, where $\mathbf{B}_{q_{4}}$ are the rows of $\mathbf{B}$ corresponding to monomials $q_{4} \mathbf{x}_{t-\ell-1}$. 
TABLE I

$\operatorname{dim} \pi^{\ell}\left(F^{(t)}\right)$ FOR SYSTEM 11

\begin{tabular}{|l|cccccc|}
\hline & $\mathrm{t}=4$ & $\mathrm{t}=5$ & $\mathrm{t}=6$ & $\mathrm{t}=7$ & $\mathrm{t}=8$ & $\mathrm{t}=9$ \\
\hline$\ell=0$ & 40 & 52 & 59 & 63 & 66 & 70 \\
$\ell=1$ & 25 & 39 & 45 & 47 & 48 & 50 \\
$\ell=2$ & 13 & 25 & 33 & 33 & $\mathbf{3 2}$ & 32 \\
$\ell=3$ & 5 & 13 & 25 & 33 & $\mathbf{3 2}$ & $\mathbf{3 2}$ \\
$\ell=4$ & 1 & 5 & 13 & 25 & 32 & 32 \\
$\ell=5$ & 0 & 1 & 5 & 13 & 25 & 32 \\
$\ell=6$ & 0 & 0 & 1 & 5 & 13 & 25 \\
$\ell=7$ & 0 & 0 & 0 & 1 & 5 & 13 \\
$\ell=8$ & 0 & 0 & 0 & 0 & 1 & 5 \\
$\ell=9$ & 0 & 0 & 0 & 0 & 0 & 1 \\
\hline
\end{tabular}

5) Compute the eigenvectors $\boldsymbol{\xi}_{i}$ of $\mathbf{M}_{q_{4}}$, and recover all solutions from the elements of $\mathbf{U}_{1} \boldsymbol{\xi}_{\boldsymbol{i}}$. The elements of this vector correspond to monomials in $\mathbf{x}_{t-\ell-1}$ evaluated at the solutions after proper scaling.

For System 11, the involutive condition is met at total degree $t=8$ and projection order $\ell=2$. As shown in Table I, the number of solutions is 32. Since using quaternions introduces double solutions, the actual number of solutions for the relative pose is 16 . The size of the expanded coefficient matrix $\mathbf{M}_{8}$ is $560 \times 495$.

Both Systems 12 and 13 reach the involutive form at total degree $t=8$, and they both have 5 variables, therefore the expanded coefficient matrices are both of size $1470 \times 1287$. However, System 12 reaches the involutive form at projection order $\ell=3$ and has 16 solutions, while System 13 is involutive at $\ell=1$ and has 28 solutions for the relative pose.

\section{Simulation Results}

We have evaluated the performance of our algorithms in simulation for different values of inter-robot measurementnoise variance, but omit tests on noise in the robots' egomotion estimates due to space limitations. Simulation results with both inter-robot and ego-motion measurement noise are presented in our technical report [16].

For our simulations, we generate random robot trajectories, with the two robots starting at initial positions $1 \mathrm{~m} \sim 2$ $\mathrm{m}$ apart from each other, and moving on an average of $3 \mathrm{~m} \sim$ $6 \mathrm{~m}$ between taking distance and/or bearing measurements. We perturb the true bearing direction to generate the bearing measurements. The perturbed bearing vectors are uniformly distributed in a cone with the true bearing as its main axis. The angle between the true vector and the boundary of the cone is defined as $\sigma_{b}$. The noise in the distance measurement is assumed zero-mean white Gaussian with standard deviation $\sigma_{d}=10 \sigma_{b} \mathrm{~m}$.

We conduct Monte Carlo simulations for different values of $\sigma_{b}$, and report the averaged results of 1000 trials per setting for each system. We report the error in position as the 2-norm of the difference between the true and the estimated position $^{6}$. To evaluate the error in the relative orientation,

\footnotetext{
${ }^{6}$ Since we focus on assessing the accuracy of the minimal problem solver, out of the multiple solutions, we choose as estimate the one closest to the true value. In practice, additional measurements are used to uniquely determine the unique position [8].
}

we use a multiplicative error model for the quaternion corresponding to the rotation matrix. In particular, true orientation, $\bar{q}$, estimated orientation, $\hat{\bar{q}}$, and error quaternion, $\delta \bar{q}$ are related via [18]:

$$
\bar{q}=\delta \bar{q} \otimes \hat{\bar{q}}
$$

where $\delta \bar{q}$ describes the small rotation that makes the estimated and the true orientation coincide. Using the smallangle approximation, the error quaternion can be written as

$$
\delta \bar{q} \simeq\left[\begin{array}{ll}
\frac{1}{2} \delta \boldsymbol{\theta}^{T} & 1
\end{array}\right]^{T} \Leftrightarrow \mathbf{C} \simeq\left(\mathbf{I}_{3}-\lfloor\delta \boldsymbol{\theta} \times\rfloor\right) \hat{\mathbf{C}}
$$

and the 2-norm of $\delta \boldsymbol{\theta}$ is used to evaluate the orientation error.

Fig. 4 shows the orientation and position errors as functions of the bearing noise $\sigma_{b}$. The curves depict the median of the error in the 1000 trials, and the vertical bars show the 25 and 75 percentiles. As expected, the error increases as the noise increases, while in the absence of measurement noise, we can recover the relative pose perfectly. We also see that the 75 percentiles are growing much faster than the 25 percentiles for most systems except for the position error of Systems 6, 7, and 10. This indicates that the probability of having larger error in the relative pose estimate increases fast with the variance of the measurement noise. The distribution of the position errors of Systems 6, 7, and 10 remains almost the same except for high noise, because it is directly measured from the distance $d_{12}$ and bearing $\mathbf{b}_{1}$. Furthermore, systems with more bearing measurements achieve better orientation accuracy than systems with more distance measurements. In particular, Systems 10 and 13 perform significantly worse than the other two systems in their groups [see Figs. 4(b) and 4(c)].

At this point, we should note that the relative pose errors will be larger when also considering noise in the robots' ego-motion estimates, especially when the robots' poses are estimated by dead-reckoning. However, the minimal solvers are practical, because they only require a small number of time steps during which the ego-motion errors will usually remain small. Finally, we note that in all cases the solutions of the minimal problems should be used in conjunction with RANSAC to perform outlier rejection followed by nonlinear weighted least squares so as to improve the estimation accuracy using all available measurements.

\section{CONCLUSION AND FUTURE WORK}

In this paper, we address the problem of computing relative robot-to-robot 3D translation and rotation using inter-robot measurements and robot ego-motion estimates. In our previous work [1], we have shown that there exist 14 base minimal systems, which result from all possible combinations of interrobot measurements, and presented closed-form algebraic solutions to three of them (Systems 1, 2, and 5 in Fig. 2). This paper addresses the remaining problems. In particular, we solve Systems 6 and 7 in closed-form, Systems 8, 9, and 10 using the Macaulay resultant, and Systems 11, 12, and 13 by employing a symbolic-numeric algorithm. These methods are significantly faster than numerical-only approaches, such as homotopy continuation [19], because they do not require iterations. 


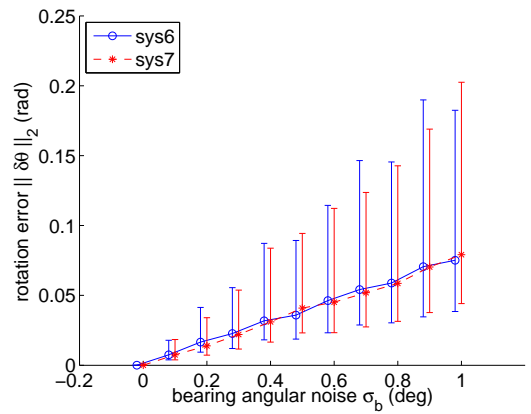

(a) Sys 6 and 7: Orientation error

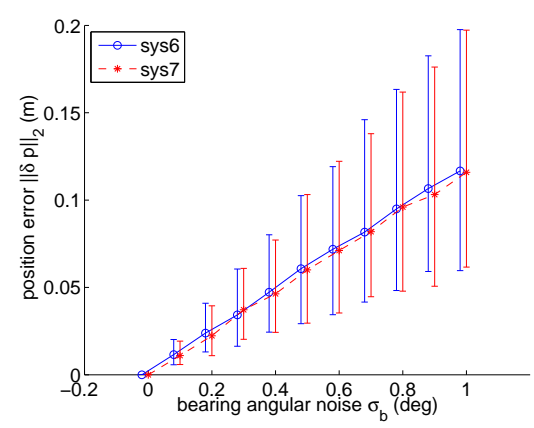

(d) Sys 6 and 7: Position error

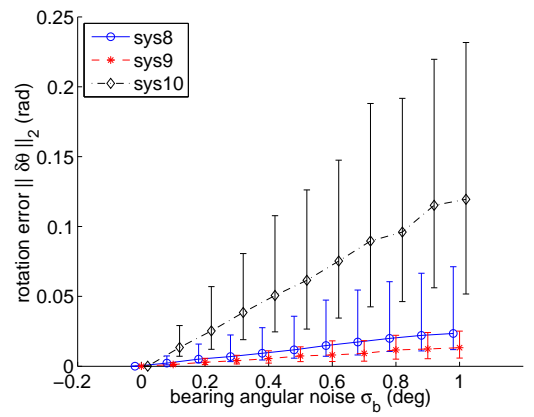

(b) Sys 8, 9, and 10: Orientation error

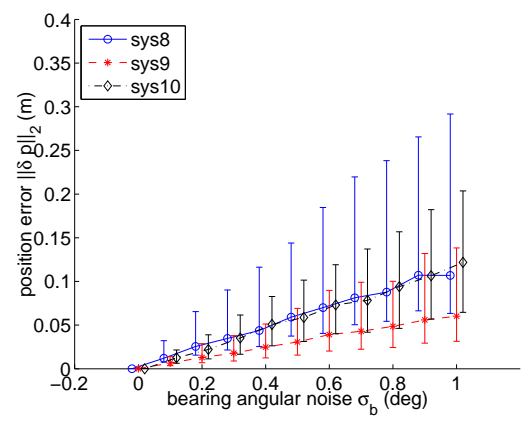

(e) Sys 8, 9, and 10: Position error

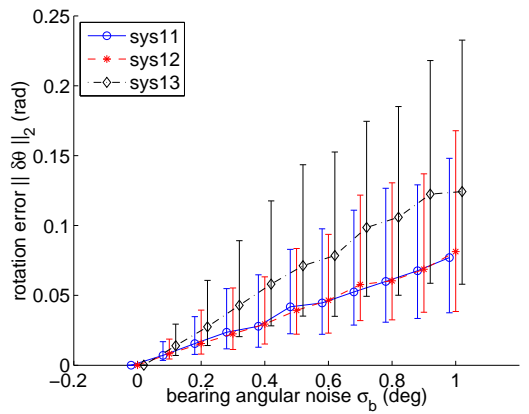

(c) Sys 11, 12 and 13: Orientation error

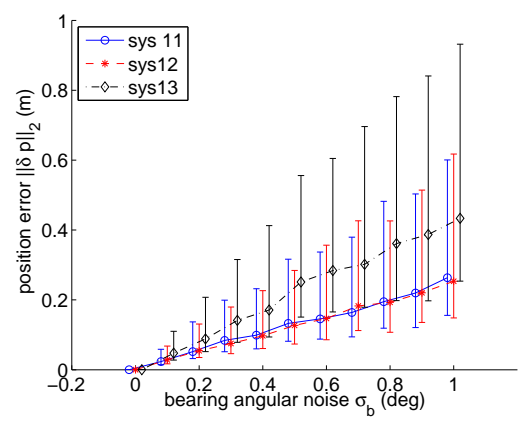

(f) Sys 11, 12, and 13: Position error

Fig. 4. Orientation and position errors as functions of the bearing-measurement noise. The plots show the median and $25-75$ percentiles in 1000 trials.

As part of our future work, we plan to optimize the robots' motion such that the uncertainty in the robot-to-robot transformation is minimized. Specifically, our objective is to determine the sequence of locations where the robots will collect the most informative measurements and thus achieve the desired level of accuracy in minimum time.

\section{REFERENCES}

[1] X. S. Zhou and S. I. Roumeliotis, "Determining the robot-to-robot 3D relative pose using combinations of range and bearing measurements: 14 minimal problems and closed-form solutions to three of them," in Proceedings of the IEEE/RSJ International Conference on Intelligent Robots and Systems, Taipei, Taiwan, Oct. 18-22, 2010, pp. 2983-2990.

[2] L. Doherty, K. S. J. Pister, and L. E. Ghaoui, "Convex position estimation in wireless sensor networks," in Proceedings of INFOCOM $20^{\text {th }}$ Annual Joint Conference of IEEE Computer and Communications Society, Anchorage, AK, Apr. 22-26, 2001, pp. 1655-1663.

[3] C.-H. Ou and K.-F. Ssu, "Sensor position determination with flying anchors in three-dimensional wireless sensor networks," IEEE Transactions on Mobile Computing, vol. 7, no. 9, pp. 1084-1097, Sept. 2008.

[4] S. Higo, T. Yoshimitsu, and I. Nakatani, "Localization on small body surface by radio ranging," in Proceedings of the 16th AAS/AIAA Space Flight Mechanics Conference, Tampa, FL, Jan. 22-26, 2006.

[5] A. Martinelli and R. Siegwart, "Observability analysis for mobile robot localization," in Proceedings of the IEEE/RSJ International Conference on Intelligent Robots and Systems, Edmonton, Canada, Aug. 2-6, 2005, pp. 1471-1476.

[6] X. S. Zhou and S. I. Roumeliotis, "Multi-robot SLAM with unknown initial correspondence: The robot rendezvous case," in Proceedings of the IEEE/RSJ International Conference on Intelligent Robots and Systems, Beijing, China, Oct. 9-15, 2006, pp. 1785-1792.

[7] A. Howard, L. E. Parker, and G. S. Sukhatme, "Experiments with a large heterogeneous mobile robot team: Exploration, mapping, deployment and detection," International Journal of Robotics Research, vol. 25, no. 5-6, pp. 431-447, May 2006.

[8] X. S. Zhou and S. I. Roumeliotis, "Robot-to-robot relative pose estimation from range measurements," IEEE Transactions on Robotics, vol. 24, no. 6, pp. 1379-1393, Dec. 2008.
[9] D. Stewart, "A platform with six degrees of freedom: A new form of mechanical linkage which enables a platform to move simultaneously in all six degrees of freedom developed by elliott-automation," Aircraft Engineering and Aerospace Technology, vol. 38, no. 4, pp. 30-35, 1993.

[10] C. W. Wampler, "Forward displacement analysis of general six-inparallel SPS (Stewart) platform manipulators using soma coordinates," Mechanism and Machine Theory, vol. 31, no. 3, pp. 331-337, Apr. 1996.

[11] T.-Y. Lee and J.-K. Shim, "Improved dialytic elimination algorithm for the forward kinematics of the general Stewart-Gough platform," Mechanism and Machine Theory, vol. 38, no. 6, pp. 563-577, June 2003.

[12] N. Trawny, X. S. Zhou, and S. I. Roumeliotis, "3D relative pose estimation from six distances," in Proceedings of Robotics: Science and Systems, Seattle, WA, June 28 - July 1, 2009, pp. 233-240.

[13] N. Trawny, X. S. Zhou, K. X. Zhou, and S. I. Roumeliotis, "Interrobot transformations in 3D," IEEE Transactions on Robotics, vol. 26, no. 2, pp. 225-243, Apr. 2010.

[14] D. A. Cox, J. Little, and D. O'Shea, Using Algebraic Geometry, 2nd ed. Springer, 2004

[15] G. Reid and L. Zhi, "Solving polynomial systems via symbolicnumeric reduction to geometric involutive form," Journal of Symbolic Computation, vol. 44, no. 3, pp. 280-291, Mar. 2009.

[16] X. S. Zhou and S. I. Roumeliotis, "Determining the robot-to-robot relative pose using range and/or bearing measurements," University of Minnesota, Dept. of Comp. Sci. \& Eng., MARS Lab, Tech. Rep., Feb. 2010. [Online]. Available: www.cs.umn.edu/ zhou/paper/14systech.pdf

[17] H. H. Chen, "Pose determination from line-to-plane correspondences: existence condition and closed-form solutions," IEEE Transactions on Pattern Analysis and Machine Intelligence, vol. 13, no. 6, pp. 530-541, June 1991.

[18] N. Trawny and S. I. Roumeliotis, "Indirect Kalman filter for 3D attitude estimation," University of Minnesota, Dept. of Comp. Sci. \& Eng., Tech. Rep. 2005-002, Jan. 2005.

[19] J. Verschelde, "Algorithm 795: PHCpack: A general-purpose solver for polynomial systems by homotopy continuation," ACM Transactions on Mathematical Software, vol. 25, no. 2, pp. 251-276, 1999. 